\title{
Sur une consultation nationale...
}

L a question des rapports sciencesociété est décidément plus que jamais à l'ordre du jour en France. C'est elle qui est au cœur de la "Consultation nationale sur les grands objectifs de la recherche française" qu'a lancée le ministre de l'Enseignement Supérieur et de la Recherche.

Alors même que près de la moitié des corps des chercheurs et enseignantschercheurs devra être renouvelée au cours des dix prochaines années, et que la crise économique actuelle conduit à une révision à la baisse des budgets de la recherche publique, cette consultation s'explique tout d'abord par des raisons politiques. Certaines d'ordre général ; d'autres plus conjoncturelles, et d'ailleurs non directement liées aux grands objectifs de la recherche française, comme la nécessité de faire face à la croissance rapide de la population étudiante.

Mais elle renvoie aussi à des interrogations plus profondes.

Il en est qui ne sont pas nouvelles, même si la conjoncture actuelle leur donne un caractère plus aigu. Ainsi, ce n'est évidemment pas un hasard si l'accent est autant mis sur la nécessité pour la recherche de répondre aux "attentes" en ce qui concerne la "lutte contre le chômage" et "I'amélioration de la compétitivité des entreprises".
D'autres par contre sont d'un ton plus nouveau et c'est la légitimité de la science qui est en cause, "la croyance au progrès engendrée par la science ne (constituant) plus une évidence pour tous ». Santé, environnement, risques naturels et technologiques occupent une place de choix dans les préoccupations quotidiennes des "citoyens" qui requièrent, selon les rapporteurs, une "recherche socialement impliquée $"$.

II y a là un certain nombre d'idées qui sont familières aux lecteurs de Natures, Sciences, Sociétés. Que " la science (soit) dans la société "s et qu'elle doive se considérer comme telle, qu'à ce titre, elle se doive en particulier « de ne pas laisser la connaissance scientifique, ses applications techniques, ses utilisations politiques, civiles ou militaires, ses conditions de création et de contrôle, en dehors de sa propre réflexion, de ses études et de ses analyses critiques ", qu'elle se perçoive comme culture et en accepte les conséquences, " toute culture (reposant) sur un certain contrôle exercé par la société elle-même ", que la prise en compte de la "complexité" soit un des défis majeurs auxquels elle ait à répondre pour aider " à résoudre les problèmes auxquels (la société) est confrontée ", voilà qui rejoint aussi sans le moindre doute notre projet éditorial...
Encore faut-il que les voies et les moyens d'une telle ambition soient bien définis. Et sur ces perspectives, le rapport d'orientation reste pratiquement totalement muet, laissant ainsi la porte ouverte à toutes les interprétations possibles sur les intentions exactes de ses promoteurs. D'où la réaction d'une partie importante de la communauté scientifique sous la forme d'un "Manifeste pour la recherche". Le débat national n'a apporté guère plus de précisions. Un vide est ainsi ouvert qui peut prêter à toutes les équivoques, l'exemple anglais, qui s'inspire de prémisses tout à fait comparables, n'étant pas fait pour rassurer!

II faut dire et redire que l'ambition de situer la science à la place qui paraît devoir être la sienne dans la société contemporaine, ne passe pas simplement par la participation des scientifiques à la " gestion des connaissances ", que ce soit à travers la formation, la valorisation, l'expertise ou la communication; elle nécessite un patient travail de la recherche sur elle-même, d'innovation dans ses démarches, ses méthodes, son organisation : en un mot une véritable recherche fondamentale. Sans cet investissement, sans cette reconnaissance, on ouvre les portes à toutes les confusions et à tous les échecs. 